\title{
Variations in patient safety climate and perceived quality of collaboration between professions in out-of-hours care
}

This article was published in the following Dove Press journal:

Journal of Multidisciplinary Healthcare

9 November 2017

Number of times this article has been viewed

\author{
Zalika Klemenc-Ketis ${ }^{1-3}$ \\ Ellen Tveter Deilkås ${ }^{4}$ \\ Dag Hofoss ${ }^{5}$ \\ Gunnar Tschudi Bondevik 6,7 \\ 'Department of Family Medicine, \\ Faculty of Medicine, University of \\ Maribor, Maribor, ${ }^{2}$ Department of \\ Family Medicine, Faculty of Medicine, \\ University of Ljubljana, Ljubljana, \\ ${ }^{3}$ Community Health Centre Ljubljana, \\ Ljubljana, Slovenia; ${ }^{4}$ Health Services \\ Research Unit, Akershus University \\ Hospital, Lørenskog, ${ }^{5}$ Institute of \\ Health and Society, University of Oslo. \\ Oslo, ${ }^{6}$ Department of Global Public \\ Health and Primary Care, University \\ of Bergen, Bergen, ${ }^{7}$ National Centre \\ for Emergency Primary Health Care, \\ Uni Research Health, Bergen, Norway
}

Correspondence: Zalika Klemenc-Ketis Department of Family Medicine, Faculty of Medicine, University of Maribor, Taborska 8, 2000 Maribor, Slovenia $\mathrm{Tel}+38641516067$ Email zalika.klemenc@um.si
Purpose: To get an overview of health care workers perceptions of patient safety climates and the quality of collaboration in Slovenian out-of-hours health care (OOHC) between professional groups.

Materials and methods: This was a cross-sectional study carried out in all (60) Slovenian OOHC clinics; 37 (61.7\%) agreed to participate with 438 employees. The questionnaire consisted of the Slovenian version of the Safety Attitudes Questionnaire - Ambulatory Version (SAQ-AV). Results: The study sample consisted of $175(70.0 \%)$ physicians, nurse practitioners, and practice nurses. Practice nurses reported the highest patient safety climate scores in all dimensions. Total mean (standard deviation) SAQ-AV score was 60.9 15.2 . Scores for quality of collaboration between different professional groups were high. The highest mean scores were reported by nurse practitioners on collaboration with practice nurses $(4.4 \pm 0.6)$. The lowest mean scores were reported by practice nurses on collaboration with nurse practitioners (3.8 \pm 0.9$)$.

Conclusion: Due to large variations in Slovenian OOHC clinics with regard to how health care workers from different professional backgrounds perceive safety culture, more attention should be devoted to improving the team collaboration in OOHC. A clearer description of professional team roles should be provided.

Keywords: patient care management, out-of-hours medical care, primary health care, patient safety

\section{Introduction}

Teamwork is defined as an interaction within a group of individuals working together to produce or deliver services for which they are mutually held accountable. ${ }^{1}$ Wellfunctioning teamwork has many advantages including promoting quality, opportunities for learning, increased efficiency, exchange of ideas, more thorough comprehensiveness, and more effective coordination and monitoring., ${ }^{2,3}$

Teamwork in health care is supposed to provide the best and most cost-effective outcomes for patients. Teams that score high on the quality of their teamwork have been reported to reduce hospitalization time and costs, improve services provision, and enhance patient satisfaction, staff motivation, and team innovation. ${ }^{2,4}$

Teamwork is also very important in primary care as health care providers with different professional backgrounds are engaged in the management of patients. ${ }^{5}$ This is also true in out-of-hours health care (OOHC) settings; here, close cooperation between different professions is needed. ${ }^{6}$ In Slovenia, OOHC clinics are organized as a part of the primary health care public network within health care centers. They 
combine $\mathrm{OOHC}$ and emergency services and are open roundthe-clock. OOHC teams consist of a physician, a practice nurse, a nurse practitioner, and other health care workers (i.e. laboratory technicians and radiology technicians). ${ }^{6}$

Patient safety culture is defined as the attitudes and behaviors that are related to patient safety and which are expected and appropriate in order to promote patient safety. ${ }^{7}$ Studies have shown that the nurse-physician collaboration is of high importance and is a critical component in the delivery of safe patient care.$^{8-10}$ The implementation of quality management systems has also been shown to be conducive to teamwork and a safety climate. ${ }^{9}$ There is evidence that improved safety climate and teamwork are associated with decreases in both patient harm and hospital mortality. ${ }^{11}$ Several educational interventions on the subject of teamwork have been shown to be effective in increasing the level of patient safety in various health care settings. ${ }^{12,13}$

Teamwork climate has been recognized as a key patient safety factor in OOHC. ${ }^{14-16}$ A recent study in Norwegian primary care reported differences in the patient safety climate in general practice and $\mathrm{OOHC}$ settings. This study also presented significant variations in perceptions of patient safety climates across different professional groups. ${ }^{14}$ In OOHC, the quality of performance seems to be associated with teamwork quality. ${ }^{17,18}$ In Slovenian OOHC clinics, communication proved to be one of the patient safety culture factors. ${ }^{16}$ This has also been pointed out in other studies. ${ }^{10,18,19}$

Perceptions of patient safety climates and quality of collaboration have not previously been studied in Slovenian OOHC clinics. However, quality teamwork and a proper safety culture are crucial for safe and high-quality health care. Therefore, we wanted to present the perceptions of patient safety climate and quality of collaboration in Slovenian OOHC clinics and identify possible variations across professional groups. This would enable us to identify good practices and detect problematic areas. Based on the results, recommendations for improvement could be made to enhance quality and safety in Slovenian $\mathrm{OOHC}$, locally and nationally. This research could also stimulate further studies in this field in Slovenia at different levels of health care.

\section{Materials and methods Type of study and settings}

This was a cross-sectional study that took place in Slovenian OOHC clinics from March 16 to May 1, 2015. The research was part of an international study led by a coordinating research group from Norway, entitled Patient Safety Culture in European Out-of-Hours Services (SAFE-EUR-OOH). The international study was part of a project by the European Research Network for Out-Of-Hours Primary Health Care (EurOOHnet), ${ }^{20}$ with several network member countries (Norway, the Netherlands, Italy, and Slovenia) performing the study. Leading researchers in these countries are members of the EurOOHnet. In Slovenia, there are 60 OOHC clinics. All were invited and 37 (61.7\%) agreed to participate. Participation was voluntary and anonymous. The respondents consented to participate by completing and sending the questionnaire. The study was approved by the National Ethics Committee of the Republic of Slovenia (No 25/11/14).

\section{Participants}

The invited employees $(n=438)$ were all members of staff (physicians, nurse practitioners, nurse managers, trainees, practice nurses, radiology technicians, and office managers) in the 37 OOHC clinics. Their participation was voluntary and anonymous. In this study, nurse practitioners are nurses with a bachelor degree - sometimes also called registered practice nurses. Practice nurses are nurses with baccalaureate education degree. ${ }^{21}$ Nurse practitioners and practice nurses differ according to the level of education and competencies. Nurse practitioners are more independent in their work, can organize and lead the health care teams, perform diagnostic and therapeutic programs, and cooperate in decision making during patient management. Practice nurses have limited responsibility regarding decision making, can participate in health care teams, prepare patients for diagnostic and therapeuric interventions, and perform health education.

\section{Data collection}

The questionnaire consisted of the Slovenian version of the Safety Attitudes Questionnaire - Ambulatory Version (SAQ-AV) that has been validated on a sample of health care employees in Slovenian OOHC. ${ }^{16}$ The SAQ-AV is a 62 -item questionnaire, where the respondents rate their agreement using a five-point Likert scale: $1=$ disagree strongly, $2=$ disagree slightly, $3=$ neutral, $4=$ agree slightly, 5= agree strongly. ${ }^{15}$ The Slovenian version of the SAQ-AV described five factors: perceptions of management, job satisfaction, safety climate, teamwork climate, and communication. ${ }^{16}$ The factor score for each individual respondent was calculated using the following formula: ${ }^{22}$

$$
=[(\Sigma \text { items }) * 100 /(5 * \mathrm{~N} \text { of items })] * 1.25-25
$$

For each factor, the minimum total score is 0 and the maximum is 100 points. 
The SAQ-AV also includes questions about the quality of collaboration with different professional groups working in the OOHC team. These questions had to be answered on a five-point Likert scale (very low, low, adequate, high, and very high).

Additionally, we surveyed demographic data (sex, age, function, and work experience). The key national researcher for Slovenia (ZKK) collected the e-mail addresses of all the willing employees in the participating OOHC clinics in Slovenia. On March 16, the electronic questionnaire was mailed to all $(n=438)$ the Slovenian health care providers that had agreed to receive an invitation to participate. The Slovenian SAQ-AV was sent from the coordinating research group in Norway, using the data collection program Qualtrics. An automatic reminder was sent to those who had not responded after two weeks. Qualtrics is an electronic software that sends links to the questionnaire to the email addresses of the participants. Participants used the link to open the online questionnaire. Their anonymous responses were stored in a database.

\section{Statistical analysis}

In the statistical analysis of this study, we included only the responses by physicians, nurse practitioners, and practice nurses. We excluded 48 participants due to incomplete questionnaires and 27 participants of other professional groups (radiologists, caregivers, and administrators). They were excluded because their number was too small to be able to make comparisons and because we wanted to compare nurses and physicians.
We used SPSS v.22.0 for the statistical analysis. One-way analysis of variance (ANOVA) with Bonferroni correction was performed to detect significant differences between physicians, nurse practitioners, and practice nurses. Bonferroni correction was used to adjust probability values for multiple comparisons. ${ }^{23}$

The observed variables used in the statistical analysis were scores on the quality of collaboration with different professional groups working in the OOHC team and scores of SAQ-AV (total and individual dimensions). The explanatory variables used in the statistical analysis were demographic characteristics. We regarded $p<0.05$ as an indicator of statistical significance.

\section{Results \\ Demographic characteristics}

Out of the 438 invited participants, 250 answered the questionnaire (57.1\% response rate). Of these, 175 (70.0\%) were physicians, nurse practitioners, and practice nurses (Table 1).

\section{Perceptions of collaboration}

The overall quality of collaboration between different professional groups was perceived as high by the respondents; mean score (standard deviation) was $4.1 \pm 0.8$. The highest quality of teamwork collaboration was reported between members of the same profession. Across different professional groups, the highest quality was reported by nurse practitioners for their collaboration with practice nurses (4.2 \pm 0.7$)$. Interestingly, the lowest quality was reported by practice nurses for their collaboration with nurse practitioners (3.8 \pm 0.9$)$ (Table 2).

Table I Demographic characteristics of the out-of-hours health care clinics' employees $(\mathrm{N}=175)$ who participated in the study, Slovenia, 2015

\begin{tabular}{|c|c|c|c|}
\hline \multirow[t]{2}{*}{ Characteristics } & Physicians ( $\mathrm{N}=93)$ & Nurse practitioners $(\mathrm{N}=43)$ & Practice nurses $(\mathrm{N}=39)$ \\
\hline & \multicolumn{3}{|l|}{ Number (\%) } \\
\hline \multicolumn{4}{|l|}{ Sex } \\
\hline Male & $30(14.9)$ & $26(14.9)$ & $19(10.9)$ \\
\hline Female & $62(30.8)$ & $17(9.8)$ & $20(11.5)$ \\
\hline \multicolumn{4}{|l|}{ Age (years) } \\
\hline$\leq 30$ & $8(4.6)$ & $10(5.7)$ & I5 (8.6) \\
\hline $31-40$ & $33(19.0)$ & $19(10.9)$ & $9(5.2)$ \\
\hline $4 I-50$ & $23(13.2)$ & $13(7.5)$ & $10(5.7)$ \\
\hline $5 I-60$ & $25(14.4)$ & I (0.6) & $4(2.3)$ \\
\hline$\geq 61$ & $3(1.7)$ & $0(0)$ & I $(0.6)$ \\
\hline \multicolumn{4}{|c|}{ Work experience (years) } \\
\hline$\leq 5$ & $19(10.9)$ & $7(4.0)$ & $7(4.0)$ \\
\hline $6-10$ & $20(11.5)$ & $5(2.9)$ & $10(5.7)$ \\
\hline $1 \mathrm{I}-20$ & $25(14.4)$ & $21(12.1)$ & $13(7.5)$ \\
\hline $21-30$ & $22(12.6)$ & $9(5.2)$ & $7(4.0)$ \\
\hline $31-40$ & $6(3.4)$ & $\mathrm{I}(0.6)$ & $\mathrm{I}(0.6)$ \\
\hline$\geq 41$ & $0(0)$ & $0(0)$ & $\mathrm{I}(0.6)$ \\
\hline
\end{tabular}




\section{Safety culture perceptions}

The mean value \pm standard deviation of the perceptions of safety culture by the participants was $58.8 \pm 13.2$ points. Practice nurses perceived safety culture the highest $(60.9 \pm 15.2)$ compared to other professional groups, but the differences between groups were not significant. Teamwork climate was the dimension perceived as the highest of all the dimensions by all professional groups (73.6 \pm 13.7$)$, followed by safety climate (63.6 \pm 18.9 ), perceptions of management (54.1 \pm 18.7 ), communication (53.0 \pm 20.9$)$, whereas job satisfaction was perceived as the lowest $(50.4 \pm 14.6)$. There were significant differences in the perceptions of management $(p=0.049)$ and safety climate scores $(p=0.006)$ across the professional groups. Perceptions of management was scored significantly lower by nurse practitioners than by physicians and practice nurses, whereas physicians scored safety climate significantly lower than practice nurses and nurse practitioners (Table 3 ).

\section{Discussion}

This is the first study in Slovenia to address variations in patient safety climate and perceived quality of collaboration between professional groups in OOHC settings. We found that, in general, the quality of team collaboration and communication in Slovenian OOHC clinics was perceived as high by the members of those teams. The highest quality of teamwork collaboration was reported between the members of the same profession. Nurse practitioners reported significantly lower quality of collaboration with physicians and practice nurses than within their own group. Total safety culture scored highest among the practice nurses. The safety climate dimension was scored significantly lower by the physicians than by the practice nurses and nurse practitioners.

In Slovenia, as elsewhere in Europe, teamwork and interprofessional collaboration is becoming increasingly more important, also as a topic for research. ${ }^{24-26}$ Teamwork is a very important basis for quality and the safe management of patients. However, merely working together does not imply good teamwork. Good and effective teamwork is based on several principles: ${ }^{27}$ positive leadership and management attributes; communication strategies and structures; personal rewards, training and development; appropriate resources and procedures; appropriate skill mix; supportive team climate; individual characteristics that support interdisciplinary team work; clarity of vision; quality and outcomes of care; and respecting and understanding roles.

In our study, members of the same profession reported very good collaboration within the group. It is common for employees with the same professional background to collaborate well with one another. However, this could also be a sign of low quality of teamwork, as it can imply an unclear vision of teamwork and problems in understanding the roles and competencies of other team members. ${ }^{27,28}$ This could

Table 2 Quality of collaboration a and communication between out-of-hours health care clinics' employees ( $N=175)$ who participated in the study; Slovenia, 2015

\begin{tabular}{lllll}
\hline Quality of collaboration & \multicolumn{1}{l}{ Physicians } & Nurse practitioners & Practice nurses & $\mathbf{p}^{\mathbf{b}}$ \\
\cline { 2 - 5 } & \multicolumn{1}{l}{ Mean (standard deviation) score } & & \\
\hline With physicians & $4.1(0.8)$ & $3.9(0.9)$ & $4.0(0.8)$ & 0.713 \\
With nurse practitioners & $3.9(0.9)$ & $4.4(0.6)$ & $3.8(0.9)$ & 0.005 \\
With practice nurses & $4.1(0.7)$ & $4.2(0.7)$ & $4.3(0.7)$ & 0.399 \\
\hline
\end{tabular}

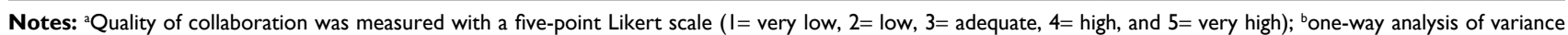
(ANOVA) with Bonferroni correction.

Table 3 Safety culture perceptions ${ }^{\mathrm{a}}$ by physicians, nurse practitioners, and practice nurses employed in $\mathrm{OOHC}$ clinics $(\mathrm{N}=175)$ who participated in the study; Slovenia, 2015

\begin{tabular}{|c|c|c|c|c|}
\hline \multirow[t]{2}{*}{ Safety culture dimension } & Physicians & Nurse practitioners & Practice nurses & $p^{b}$ \\
\hline & \multicolumn{4}{|c|}{ Mean (standard deviation) score } \\
\hline Perceptions of management & $54.6(18.6)$ & $48.7(18.8)$ & $58.9(18.7)$ & 0.049 \\
\hline Job satisfaction & $50.8(14.8)$ & $48.2(13.8)$ & $52.1(15.1)$ & 0.468 \\
\hline Safety climate & $57.9(19.2)$ & $63.5(19.5)$ & $69.5(18.1)$ & 0.006 \\
\hline Teamwork climate & $76.4(11.5)$ & $73.1(15.5)$ & $71.4(14.3)$ & 0.136 \\
\hline Communication & $55.2(21.4)$ & $50.8(18.8)$ & $52.9(22.5)$ & 0.521 \\
\hline Total SAQ-AV score & $59.0(12.7)$ & $56.7(11.7)$ & $60.9(15.2)$ & 0.337 \\
\hline
\end{tabular}

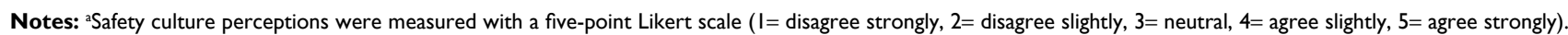

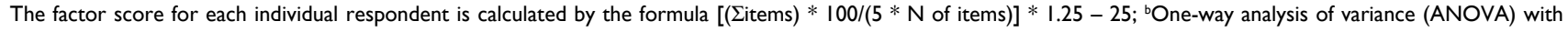
Bonferroni correction.

Abbreviation: OOHC, out-of-hours health care; SAQ, Safety Attitudes Questionnaire - Ambulatory Version. 
result in unhealthy competition, focusing on different goals, negative stereotyping, and hierarchical communication. ${ }^{25}$

Nurse practitioners reported that their collaboration with physicians was low. Recently, nursing roles have expanded and formalized and have come to be regarded as more autonomous. This could present a threat to the autonomy of other professional groups due to unclear distribution of roles and competencies leading to disputes between physicians and nurses. ${ }^{26,29}$ This was shown in another study from Slovenia on teamwork between nurses and physicians. ${ }^{30}$ That study observed problems in teamwork in terms of hierarchy, lack of cooperation and team building, and insufficient collaboration between both professional groups in implementing changes. This is in accordance with the findings of our study, where communication was scored low by practice nurses and physicians. The results may indicate problems in teamwork in different health care settings in Slovenia, which could affect patient safety.

Nurse practitioners reported good collaboration with practice nurses; however, this was not the case in reverse as the lowest quality reported by practice nurses was for their collaboration with nurse practitioners. These findings indicate that serious problems exist in terms of collaboration between these professional groups. It is possible that there is a lack of open communication, which could very well be the consequence of problems in accepting the new role of nurse practitioner in primary health care in Slovenia.

The factor communication scored low in our study, similar to a study from Slovenian hospitals. ${ }^{31}$ On the other hand, our study showed that teamwork was graded as the highest safety culture dimension of all. This finding correlates with the results of a Slovenian study on ethical dilemmas in family medicine, where teamwork was not regarded as a problem. ${ }^{32,33}$ This is a somewhat contrary finding, as good communication is usually necessary for good interprofessional teamwork. However, communication in the safety field is about willingness and readiness to talk about safety issues, which is something different to teamwork. The results of our study indicate a need for improving interprofessional communication in OOHC teams with regard to safety issues. We should bear in mind that quality and safe health care depend greatly on talking about - and learning from - medical errors. ${ }^{25}$

The dimension with the lowest score was job satisfaction. This finding was not surprising, as there is a great shortage of physicians and nurses in Slovenia, and many health care providers are overburdened. ${ }^{34}$

Safety climate was perceived lower by the physicians than by the practice nurses and the nurse practitioners. A similar finding was reported by a Norwegian study in $\mathrm{OOHC}$ settings. ${ }^{35}$ This might be a consequence of the fact that nurses work only in OOHC settings, while the physicians' work there is limited because they also work in family medicine practices. Employees who spend most of their working hours in $\mathrm{OOHC}$ will probably feel more comfortable in that clinical setting. ${ }^{35}$

The factor perceptions of management was perceived lower by nurse practitioners than by practice nurses and physicians. This is in accordance with a Slovenian study on the relationships between nurses and physicians, which showed that professional growth was mainly threatened by organizational factors such as hierarchy, control orientation, lack of cooperation, and team building. ${ }^{30}$ It seems that nurse practitioners may feel some kind of threat also from the management in $\mathrm{OOHC}$ clinics.

There are different organizational models of $\mathrm{OOHC}$ in Europe. ${ }^{6}$ Additionally, national patient strategies also differ between different European countries. ${ }^{36}$ The results of this study are, therefore, unique for the Slovenian OOHC settings but, on the other hand, they add to the body of international knowledge from this field.

This study has some limitations that have to be mentioned. We aimed to include all OOHC clinics in Slovenia; however, only two-thirds $(\mathrm{N}=37)$ of them were willing to participate. Around $60 \%$ of the 438 employees in these clinics participated and filled in the questionnaire. Even though the response rates of clinics and employees were acceptable, one must be careful with generalizing the findings to the whole of Slovenia. On the other hand, the results of this study were in accordance with the findings of other similar Slovenian studies, which gives us confidence in their reliability and validity.

The following recommendations can be offered according to the study results. More attention should be devoted to improving the team collaboration in Slovenian OOHC and to improving the collaboration between physicians and nurses. Also, the collaboration of practice nurses and nurse practitioners should be further explored. Communication about safety issues should be stimulated within the OOHC team. Health authorities and managers of $\mathrm{OOHC}$ in Slovenia should engage in a clearer description of professional roles and roles in a team and reconsider their relationship with the staff.

Further studies should be made to more deeply explore safety culture in OOHC settings. More attention should be devoted to improving the collaboration between physicians and nurses in OOHC teams.

\section{Conclusion}

Large variations in Slovenian OOHC clinics were found in terms of how health care workers from different profes- 
sional backgrounds perceive safety culture. The relationship between nurses with different professional backgrounds seems to be a burning issue that requires more attention. The safety culture domains "communication" and "perceptions of management" require improvement.

\section{Acknowledgments}

The National Centre for Emergency Primary Health Care, Uni Research Health, Bergen, Norway, covered the expenses of the translation/back-translation of the Safety Attitudes Questionnaire and data collection. The authors acknowledge the financial support from the Slovenian Research Agency (research core funding number P3 0339).

\section{Disclosure}

The authors report no conflicts of interest in this work.

\section{References}

1. Mohman SA, Cohen SG, Mohrman AM. Designing Team-based Organizations. San Francisco: Jossey-Bass; 1995.

2. Borrill CS, Carletta J, Carter AJ, et al. The effectiveness of health care teams in the National Health Service. 1999. Available from: http:// homepages.inf.ed.ac.uk/jeanc/DOH-final-report.pdf. Accessed January 27, 2016.

3. Ivetic V, Pasic K, Selic P. The effect of an educational intervention in family phisicians on self-rated quality of life in patients with medically unexplained symptoms. Zdr Varst. 2017;56(2):91-98.

4. Makivić I, Kersnik J, Klemenc-Ketis Z. The role of the psychosocial dimension in the improvement of quality of care: a systematic review. Zdr Varst. 2016;55(1):86-95.

5. Poplas-Susic T, Svab I, Kersnik J. The project of model practices in family medicine in Slovenia. Zdrav Vestn. 2013;82(10):635-647.

6. Huibers L, Giesen P, Wensing M, Grol R. Out-of-hours care in Western countries: assessment of different organizational models. BMC Health Serv Res. 2009;9:105.

7. Sammer CE, James BR. Patient safety culture: the nursing unit leader's role. Online J Issues Nurs. 2011;16(3):3.

8. McComb SA, Lemaster M, Henneman EA, Hinchey KT. An evaluation of shared mental models and mutual trust on general medical units: implications for collaboration, teamwork, and patient safety. J Patient Saf. 2015. [Epub ahead of print].

9. Kristensen S, Hammer A, Bartels P, et al. Quality management and perceptions of teamwork and safety climate in European hospitals. Int J Qual Health Care. 2015;27(6):498-505.

10. Gluyas H. Effective communication and teamwork promotes patient safety. Nurs Stand. 2015;29(49):50-57.

11. Berry JC, Davis JT, Bartman T, et al. Improved safety culture and teamwork climate are associated with decreases in patient harm and hospital mortality across a hospital system. J Patient Saf. 2016. [Epub ahead of print].

12. Kirkman MA, Sevdalis N, Arora S, Baker P, Vincent C, Ahmed M. The outcomes of recent patient safety education interventions for trainee physicians and medical students: a systematic review. BMJ Open. 2015;5(5): e007705.

13. Epps HR, Levin PE. The TeamSTEPPS approach to safety and quality. J Pediatr Orthop. 2015;35(5 Suppl 1):S30-S33.

14. Bondevik GT, Hofoss D, Hansen EH, Deilkas EC. The safety attitudes questionnaire - ambulatory version: psychometric properties of the Norwegian translated version for the primary care setting. BMC Health Serv Res. 2014;14:139.
15. Sexton JB, Helmreich RL, Neilands TB, et al. The safety attitudes questionnaire: psychometric properties, benchmarking data, and emerging research. BMC Health Serv Res. 2006;3:44.

16. Klemenc-Ketis Z, Maletic M, Stropnik V, Deilkås ET, Hoffos D, Bondevik GT. The safety attitudes questionnaire - ambulatory version: psychometric properties of the Slovenian version for the out-of-hours primary care setting. BMC Health Serv Res. 2017;17:36.

17. Tourani S, Hassani M, Ayoubian A, Habibi M, Zaboli R. Analyzing and prioritizing the dimensions of patient safety culture in emergency wards using the TOPSIS technique. Glob J Health Sci. 2015;7(4): $143-150$.

18. Meier C. Importance of good teamwork in urgent care services. Emerg Nurse. 2014;22(7):32-36.

19. Ghaferi AA, Dimick JB. Importance of teamwork, communication and culture on failure-to-rescue in the elderly. Br J Surg. 2016;103(2):e47-e51.

20. Huibers L, Phillips H, Giesen P, Remmen R, Christensen MB, Bondevik GT. EurOOHnet - the European research network for out-of-hours primary health care. Eur J Gen Pract. 2014;20(3):229-232.

21. Oandasan IF, Hammond M, Conn LG, Callahan S, Gallinaro A, Moaveni A. Family practice registered nurses: the time has come. Can Fam Physician. 2010;56(10):e375-e382.

22. Baker R, Hearnshaw H. A Method for Surveying Patient Satisfaction Audit Protocol PS1. Leicester: Eli Lilly National Cinical Audit Centre; 1996.

23. Armstrong RA. When to use the Bonferroni correction. Ophthalmic Physiol Opt. 2014;34(5):502-508.

24. Petek Ster M, Ster B. The role of education for graduated nurses in model practices: example of arterial hypertension. Obzor Zdrav Neg. 2015;49(1):52-59.

25. Thomson K, Outram S, Gilligan C, Levett-Jones T. Interprofessional experiences of recent healthcare graduates: a social psychology perspective on the barriers to effective communication, teamwork, and patient-centred care. J Interprof Care. 2015;29(6):634-640.

26. Ellingson LL. Communication, collaboration, and teamwork among health care professionals. Comm Res Trends. 2002;21(3):2-15.

27. Nancarrow SA, Booth A, Ariss S, Smith T, Enderby P, Roots A. Ten principles of good interdisciplinary team work. Hum Resour Health. 2013;11:19.

28. Leggat SG. Effective healthcare teams require effective team members: defining teamwork competencies. BMC Health Serv Res. 2007;7:17.

29. Sullivan-Bentz M, Humbert J, Cragg B, et al. Supporting primary health care nurse practitioners' transition to practice. Can Fam Physician. 2010;56(11):1176-1182.

30. Skela Savic B, Pagon M. Relationship between nurses and physicians in terms of organizational culture: who is responsible for subordination of nurses? Croat Med J. 2008;49(3):334-343.

31. Robida A. Hospital survey on patient safety culture in Slovenia: a psychometric evaluation. Int J Qual Health Care. 2013;25(4): 469-475.

32. Klemenc-Ketis Z, Kersnik J, Ojstersek J. Perceived difficulties in managing ethical problems in family practice in Slovenia: cross-sectional study. Croat Med J. 2008;49:799-806.

33. Klemenc-Ketis Z, Kersnik J. Prevalence of ethical dilemmas in Slovenian family practice. Acta Medico-Biotechnica. 2009;2:47-56.

34. Selič P, Stegne-Ignjatović T, Klemenc-Ketis Z. Burnout among Slovenian family medicine trainees: a cross-sectional study. Zdrav Vestn. 2012;81:218-224.

35. Bondevik GT, Hofoss D, Holm Hansen E, Deilkas ET. Patient safety culture in Norwegian primary care: a study in out-of-hours casualty clinics and GP practices. Scand J Prim Health Care. 2014;32: $132-138$.

36. Godycki-Cwirko M, Esmail A, Dovey S, et al. Patient safety initiatives in Central and Eastern Europe: a mixed methods approach by the LINNEAUS collaboration on patient safety in primary care. Eur J Gen Pract. 2015;21(Suppl):62-68. 


\section{Publish your work in this journal}

The Journal of Multidisciplinary Healthcare is an international, peerreviewed open-access journal that aims to represent and publish research in healthcare areas delivered by practitioners of different disciplines. This includes studies and reviews conducted by multidisciplinary teams as well as research which evaluates the results or conduct of such teams or health care processes in general. The journal covers a very wide range of areas and welcomes submissions from practitioners at all levels, from all over the world The manuscript management system is completely online and includes a very quick and fair peer-review system. Visit http://www.dovepress.com/ testimonials.php to read real quotes from published authors.

Submit your manuscript here: https://www.dovepress.com/journal-of-multidisciplinary-healthcare-journal 